\title{
Tetrahydropyranylation of Alcohols and Phenols by Using Simple Simple and Inexpensive Organocatalyst
}

\author{
V. Sivarama Krishna Reddy and P. V. V. Satyanarayana* \\ (Department of Chemistry, Acharya Nagarjuna University, Nagarjuna Nagar, Guntur, India)
}

\begin{abstract}
A simple and convenient procedure for tetrahydropyranylation of alcohols and phenols in the presence of organocatalyst, 3,5-dinitrobenzoic acid, has been developed. 3,5-Dinitrobenzoic acid is an effective, very cheap and viable catalyst in above synthetic transformations with various alcohols, phenols. A large number of alcohols and phenols have been tolerated well in this protocol.
\end{abstract}

Keywords: Organocatalyst, 3,5-Dinitrobenzoic acid, Tetrahydropyranylation, alcohols, phenols.

\section{Introduction}

The choice of the protection and deprotection strategy in a synthetic series is inevitable, owing to chemoselective transformations in the presence of various functional groups. The tetrahydropyranyl ethers (THP ethers) are attractive because of low cost of 3,4-dihydro-2H-pyran (DHP) and stability of THP derivatives towards various reaction conditions such as strong bases, Grignard reagents, hydrides, redox reagents, alkylating, acylating agents and catalytic hydrogenation and easy removal under mild acidic conditions. This transformation is generally achieved using both protonic as well as Lewis acid catalysts. Consequently a variety of reagents such as pTSA, ${ }^{2} \quad \mathrm{BF}_{3} . \mathrm{OEt}_{2},{ }^{3} \mathrm{ZnCl}_{2}$ impregnated alumina, ${ }^{4}{ }^{4}$ TBATB, ${ }^{5}$ PPTS, ${ }^{6}$ acetonyltriphenylphosphonium bromide, ${ }^{7}$ aluminum chloride hexahydrate ${ }^{8} \operatorname{In}(\mathrm{OTf})_{3}{ }^{9} \mathrm{Bi}(\mathrm{OTf})_{3}{ }_{10}{ }^{10} \mathrm{LiOTf},{ }^{11}$ dialkylimidazolium tetrachloroaluminates, ${ }^{12} \mathrm{InCl}_{3}$ immoblized in ionic liquids, ${ }^{13}$ bromodimethylsulfonium bromide, ${ }^{14}$ cupric sulfate pentahydrate, ${ }^{15} \mathrm{CAN},{ }^{16}$ and bismuth(III) nitrate pentahydrate ${ }^{17}$ and photosensitization ${ }^{18}$ have been introduced for tetrahydropyranyl protection of alcohols and phenols. However, some of these procedures suffer due to the use of expensive and moisture sensitive catalysts, high temperature, longer reaction times and incompatibility with other functional groups, and some reagents also have to be freshly prepared prior to use. ${ }^{6,8,11,15}$ Though metal triflates have been found to be effective catalysts for tetrahydropyranylation, these reagents have limited applicability since they are relatively expensive, difficult to handle, and not readily available. Furthermore, the procedures involving these reagents require harsh and inert reaction conditions.

\section{Results and Discussions}

With an objective of developing a viable procedure for tetrahydropyranylation, we focused on finding a cheap and efficient catalyst that would give high yields and easy handling procedure under aerobic conditions. In continuation of our work on novel organocatalysts for organic transformations, we became interested to use very cheap 3,5-dinitrobenzoic acid (3,5-DNBA, $<\$ 0.1$ per gram) as organocatalyst for the aforementioned reaction. Though 3,5-DNBA has been used as an additive in many reactions, ${ }^{19}$ it has not been used as a catalyst. In this paper, we describe the successful implementation of 3,5-DNBA as an organocatalyst for the tetrahydropyranylation of various primary and secondary alcohols and phenols.<smiles>O=C(O)c1cc([N+](=O)[O-])cc([N+](=O)[O-])c1</smiles>

3,5-Dinitrobenzoic acid (3,5-DNBA)

Initially, the tetrahydropyranylation of phenol $(1 \mathrm{mmol})$ was performed with DHP $(1 \mathrm{mmol})$ in $\mathrm{CH}_{2} \mathrm{Cl}_{2}(5 \mathrm{~mL})$ in the presence of $20 \mathrm{~mol} \%$ 3,5-DNBA at room temperature under aerobic conditions (Table 1, Entry 7). As a result the transformation took place in $3 \mathrm{~h}$ to afford its tetrahydropyranyl ether $\mathbf{2 g}$ in $88 \%$ yield. We have also performed the reaction with $10 \mathrm{~mol} \%$ catalyst but the reaction was completed in $11 \mathrm{~h}$ with $85 \%$ yield of $\mathbf{2 g}$. Using the protocol, various primary, secondary alcohols and phenols were transformed easily into the corresponding THP-ethers in good yields (Table 1). It is observed that phenols bearing electron withdrawing substituents gave protected THP-ethers in good yields (Table 1, Entry 14). Importantly, no isomerization of double bond took place in case of eugenol (Table 1, Entry 16). 


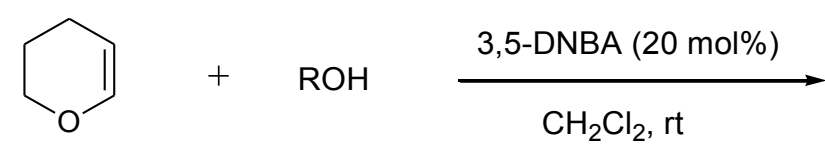

$1 \quad \mathrm{R}=$ alkyl, aryl<smiles>[R]OC1CCCCO1</smiles>

2

Table 1. Tetrahydropyranylation of alcohols and phenols catalyzed by 3,5-dinitrobenzoic acid. ${ }^{\text {a }}$

\begin{tabular}{|c|c|c|c|c|}
\hline Entry & $\begin{array}{c}\text { Substrate } \\
\text { (ROH) }\end{array}$ & $\begin{array}{c}\text { Time } \\
\text { (h) }\end{array}$ & $\begin{array}{c}\text { Product } \\
\text { (ROTHP) }\end{array}$ & Yield $^{b}(\%)$ \\
\hline 1 & $ح_{\mathrm{OH}}$ & 2 & $2 a$ & 91 \\
\hline 2 & $ح \mathrm{OH}$ & 2 & $2 \mathrm{~b}$ & 91 \\
\hline 3 & $\curvearrowright \curvearrowright_{\mathrm{OH}}$ & 2 & $2 \mathrm{c}$ & 92 \\
\hline 4 & & 2 & $2 d$ & 94 \\
\hline 5 & & 2 & $2 \mathrm{e}$ & 97 \\
\hline 6 & & 2 & $2 \mathrm{f}$ & 98 \\
\hline 7 & & 3 & $2 \mathrm{~g}$ & 88 \\
\hline 8 & & 6 & $2 \mathrm{~h}$ & 80 \\
\hline 9 & & 8 & $2 \mathrm{i}$ & 86 \\
\hline 10 & & 4 & $2 \mathrm{j}$ & 91 \\
\hline 11 & & 4 & $2 k$ & 82 \\
\hline 12 & & 4 & 21 & 91 \\
\hline 13 & & 3 & $2 m$ & 85 \\
\hline 14 & & 8 & $2 n$ & 84 \\
\hline 15 & & 8 & 20 & 80 \\
\hline
\end{tabular}




\begin{tabular}{|l|l|l|l|l|}
\hline 16 & 8 & $2 \mathbf{2 p}$ & 81 \\
\hline 17 & 4 & $2 \mathbf{2 q}$ & 81 \\
\hline 18
\end{tabular}

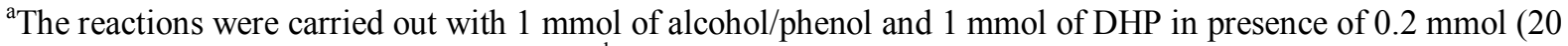
mol\%) of 3,5-DNBA at room temperature. ${ }^{b}$ Yields are of pure and isolated products.

\section{Conclusion}

In conclusion, we have developed a simple and convenient method for tetrahydropyranylation of alcohols and phenols in the presence of 3,5-dinitrobenzoic acid. 3,5-dinitrobenzoic acid is an effective, very cheap and viable catalyst in above synthetic transformations with various alcohols, phenols. We believe that this methodology is valuable addition to modern synthetic methodologies.

\section{Experimental}

\section{General procedure for the synthesis of tetrahydropyranyl ethers}

A solution of an alcohol or a phenol (1 mmol), dihydro-2H-pyran (DHP, $1 \mathrm{mmol}$ ), and 3.5dinitrobenzoic acid (3,5-DNBA, $0.2 \mathrm{mmol}, 20 \mathrm{~mol} \%)$, in $\mathrm{CH}_{2} \mathrm{Cl}_{2}(2 \mathrm{~mL})$ were stirred at ambient temperature for an appropriate time (monitored by TLC or GC). After completion of the reaction ( $45 \mathrm{~min}$ for alcohols and $3 \mathrm{~h}$ for phenols), the organic layer was washed twice with $10 \mathrm{~mL}$ of saturated $\mathrm{NaHCO}_{3}$ solution, dried (anhyd. $\mathrm{Na}_{2} \mathrm{SO}_{4}$ ), and concentrated under reduced pressure to yield almost pure product. The product was purified further by column chromatography on silica gel using ethyl acetate/hexanes as the eluent (1:9).

Prop-2-enyl tetrahydro-2H-pyran-2-yl ether (2a):

Reaction time: $45 \mathrm{~min}$.

Yield: $0.131 \mathrm{~g}(92 \%)$ as colourless oily liquid.

IR (neat) $\mathbf{v}_{\max }: 3065,2956,2834,1457,1025,1014 \mathrm{~cm}^{-1}$.

${ }^{1} \mathbf{H}$ NMR $\left(\mathbf{C D C l}_{3}, \mathbf{5 0 0} \mathbf{M H z}\right): \delta 5.97-5.89(\mathrm{~m}, 1 \mathrm{H}), 5.30-5.26(\mathrm{~m}, 1 \mathrm{H}), 5.15(\mathrm{~d}, \mathrm{~J}=10.5 \mathrm{~Hz}, \overline{1 \mathrm{H}}), 4.63(\mathrm{t}, \mathrm{J}=3$ $\mathrm{Hz}, 1 \mathrm{H}), 4.24-4.21(\mathrm{~m}, 1 \mathrm{H}), 3.99-3.95(\mathrm{~m}, 1 \mathrm{H}), 3.88-3.84(\mathrm{~m}, 1 \mathrm{H}), 3.51-3.47(\mathrm{~m}, 1 \mathrm{H}), 1.85-1.81(\mathrm{~m}, 1 \mathrm{H}), 1.74-$ $1.68(\mathrm{~m}, 1 \mathrm{H}), 1.62-1.50(\mathrm{~m}, 4 \mathrm{H}) \mathrm{ppm}$.

${ }^{13} \mathbf{C}$ NMR (CDCl 3 , 125 MHz): $\delta$ 135.1, 117.0, 98.3, 68.3, 62.5, 31.0, 25.9, 19.8 ppm.

MS: $\mathrm{m} / \mathrm{z}=142\left[\mathrm{M}^{+}\right]$.

Propyl tetrahydro-2H-pyran-2-yl ether (2b):

Reaction time: $45 \mathrm{~min}$.

Yield: $0.133 \mathrm{~g}(93 \%)$ as colourless oily liquid.

IR (neat) $\mathbf{v}_{\max }: 2943,2854,1457,1336,1101,1056 \mathrm{~cm}^{-1}$.

$\left.{ }^{1} \mathbf{H ~ N M R ~ ( C D C l}_{3}, \mathbf{5 0 0} \mathbf{M H z}\right): \delta 4.55(\mathrm{t}, \mathrm{J}=3.5 \mathrm{~Hz}, 1 \mathrm{H}), 3.86-3.82(\mathrm{~m}, 1 \mathrm{H}), 3.68-3.64(\mathrm{~m}, 1 \mathrm{H}), 3.48-3.45(\mathrm{~m}$, $1 \mathrm{H}), 3.35-3.30(\mathrm{~m}, 1 \mathrm{H}), 1.82-1.79(\mathrm{~m}, 1 \mathrm{H}), 1.70-1.66(\mathrm{~m}, 1 \mathrm{H}), 1.61-1.48(\mathrm{~m}, 6 \mathrm{H}), 0.90(\mathrm{t}, \mathrm{J}=7.5 \mathrm{~Hz}, 3 \mathrm{H}) \mathrm{ppm}$.

${ }^{13} \mathbf{C}$ NMR (CDCl $\left.\mathbf{3}, \mathbf{1 2 5} \mathbf{M H z}\right): \delta 98.8,69.2,62.3,30.7,25.4,22.9,19.6,10.6 \mathrm{ppm}$.

MS: $\mathrm{m} / \mathrm{z}=145[\mathrm{M}+\mathrm{H}]^{+}$.

Butyl tetrahydro-2H-pyran-2-yl ether (2c):

Reaction time: $45 \mathrm{~min}$.

Yield: $0.146 \mathrm{~g}(93 \%)$ as colourless oily liquid.

IR (neat) $\mathbf{v}_{\max }: 2946,2853,1452,1365,1152,1065 \mathrm{~cm}^{-1}$.

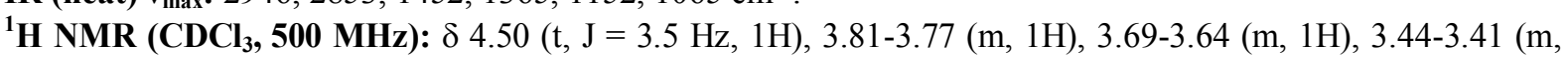
$1 \mathrm{H}), 3.33-3.29(\mathrm{~m}, 1 \mathrm{H}), 1.77-1.75(\mathrm{~m}, 1 \mathrm{H}), 1.65-1.61(\mathrm{~m}, 1 \mathrm{H}), 1.52-1.43(\mathrm{~m}, 6 \mathrm{H}), 1.34-1.30(\mathrm{~m}, 2 \mathrm{H}), 0.86(\mathrm{t}, \mathrm{J}=$ $7.5 \mathrm{~Hz}, 3 \mathrm{H}) \mathrm{ppm}$.

${ }^{13} \mathbf{C}$ NMR (CDCl 3 , 125 MHz): $\delta$ 99.1, 67.7, 62.6, 32.2, 31.1, 25.9, 20.0, 19.8, $14.2 \mathrm{ppm}$.

MS: $\mathrm{m} / \mathrm{z}=158\left[\mathrm{M}^{+}\right]$. 
Cyclohexyl tetrahydro-2H-pyran-2-yl ether (2d):

Reaction time: $45 \mathrm{~min}$.

Yield: $0.173 \mathrm{~g}(94 \%)$ as colourless oily liquid.

IR (neat) $\mathbf{v}_{\max }: 2932,2858,1451,1354,1125,1068 \mathrm{~cm}^{-1}$.

${ }^{1} \mathbf{H}$ NMR $\left(\mathbf{C D C l}_{3}, \mathbf{5 0 0} \mathbf{M H z}\right): \delta 4.72(\mathrm{bs}, 1 \mathrm{H}), 3.93-3.87(\mathrm{~m}, 1 \mathrm{H}), 3.63-3.59(\mathrm{~m}, 1 \mathrm{H}), 3.50-3.47(\mathrm{~m}, 1 \mathrm{H}), 1.91-$ $1.84(\mathrm{~m}, 3 \mathrm{H}), 1.75-1.70(\mathrm{~m}, 3 \mathrm{H}), 1.55-1.54(\mathrm{~m}, 5 \mathrm{H}), 1.40-1.18(\mathrm{~m}, 5 \mathrm{H}) \mathrm{ppm}$.

${ }^{13} \mathbf{C}$ NMR (CDCl $\mathbf{3}$, 125 MHz): $\delta$ 96.6, 74.4, 62.8, 33.7, 31.8, 31.3, 25.7, 25.5, 24.5, 24.2, 20.0 ppm.

MS: $\mathrm{m} / \mathrm{z}=184\left[\mathrm{M}^{+}\right]$.

Benzyl tetrahydro-2H-pyran-2-yl ether (2e):

Reaction time: $45 \mathrm{~min}$.

Yield: $0.184 \mathrm{~g}(96 \%)$ as colourless oily liquid.

IR (neat) $v_{\max }: 3052,2948,1675,1594,964,850,742 \mathrm{~cm}^{-1}$.

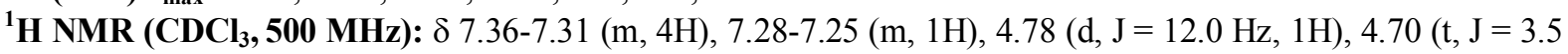
$\mathrm{Hz}, 1 \mathrm{H}), 4.49(\mathrm{~d}, \mathrm{~J}=12.0 \mathrm{~Hz}, 1 \mathrm{H}), 3.93-3.89(\mathrm{~m}, 1 \mathrm{H}), 3.56-3.51(\mathrm{~m}, 1 \mathrm{H}), 1.87-1.85(\mathrm{~m}, 1 \mathrm{H}), 1.73-1.50(\mathrm{~m}, 5 \mathrm{H})$ ppm.

${ }^{13} \mathbf{C}$ NMR (CDCl $\left.\mathbf{3}, \mathbf{1 2 5} \mathbf{M H z}\right): \delta 138.3,128.3,127.8,127.5,97.7,68.8,62.1,30.6,25.5,19.4 \mathrm{ppm}$.

MS: $\mathrm{m} / \mathrm{z}=193[\mathrm{M}+\mathrm{H}]^{+}$.

3-Phenyl-2-propenyl tetrahydro-2H-pyran-2-yl ether (2f):

Reaction time: $45 \mathrm{~min}$.

Yield: $0.207 \mathrm{~g}(95 \%)$ as colourless oily liquid.

IR (neat) $\mathbf{v}_{\max }: 3055,3023,2943,1593,1119,984,755 \mathrm{~cm}^{-1}$

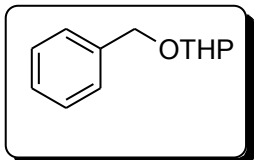

${ }^{1} \mathbf{H}$ NMR $\left(\mathbf{C D C l}_{3}, \mathbf{5 0 0} \mathbf{M H z}\right): \delta 7.38(\mathrm{~d}, \mathrm{~J}=8.0 \mathrm{~Hz}, 2 \mathrm{H}), 7.29(\mathrm{t}, \mathrm{J}=8.0 \mathrm{~Hz}, 2 \mathrm{H}), 7.22(\mathrm{t}, \mathrm{J}=8.0 \mathrm{~Hz}, 1 \mathrm{H}), 6.61$ $(\mathrm{d}, \mathrm{J}=8.0 \mathrm{~Hz}, 1 \mathrm{H}), 6.33-6.28(\mathrm{~m}, 1 \mathrm{H}), 4.70(\mathrm{t}, \mathrm{J}=3.5 \mathrm{~Hz}, 1 \mathrm{H}), 4.41-4.37(\mathrm{~m}, 1 \mathrm{H}), 4.17-4.13(\mathrm{~m}, 1 \mathrm{H}), 3.92-3.88$ $(\mathrm{m}, 1 \mathrm{H}), 3.55-3.50(\mathrm{~m}, 1 \mathrm{H}), 1.87-1.85(\mathrm{~m}, 1 \mathrm{H}), 1.76-1.72(\mathrm{~m}, 1 \mathrm{H}), 1.66-1.51(\mathrm{~m}, 4 \mathrm{H}) \mathrm{ppm}$.

${ }^{13}$ C NMR (CDCl 3 , 125 MHz): $\delta$ 136.7, 132.1, 128.4, 127.4, 126.3, 125.9, 97.7, 67.5, 62.0, 30.5, 25.3, 19.3 ppm.

MS: $\mathrm{m} / \mathrm{z}=219[\mathrm{M}+\mathrm{H}]^{+}$.

Phenyl tetrahydro-2H-pyran-2-yl ether (2g):

Reaction time: $3 \mathrm{~h}$.

Yield: $0.170 \mathrm{~g}(96 \%)$ as colourless oily liquid.

IR (neat) $\mathbf{v}_{\text {max }}: 3059,2943,1598,1589,964,920,754 \mathrm{~cm}^{-1}$.

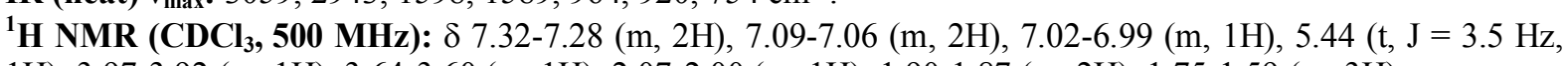
$1 \mathrm{H})$, 3.97-3.92 (m, 1H), 3.64-3.60 (m, 1H), 2.07-2.00 (m, 1H), 1.90-1.87 (m, 2H), 1.75-1.59 (m, 3H) ppm.

${ }^{13} \mathbf{C}$ NMR (CDCl 3 , 125 MHz): $\delta$ 157.0, 129.3, 121.5, 116.4, 96.2, 61.9, 30.3, 25.1, 18.7 ppm.

MS: $\mathrm{m} / \mathrm{z}=179[\mathrm{M}+\mathrm{H}]^{+}$.

2-Methoxyphenyl tetrahydro-2H-pyran-2-yl ether (2h):

Reaction time: $3 \mathrm{~h}$.

Yield: $0.191 \mathrm{~g}(92 \%)$ as colourless oily liquid.

IR (neat) $\mathbf{v}_{\max }: 3055,2943,1592,1502,1455,872,770 \mathrm{~cm}^{-1}$.

${ }^{1} \mathbf{H}$ NMR $\left(\mathbf{C D C l}_{3}, \mathbf{5 0 0} \mathbf{M H z}\right): \delta 7.02-6.99(\mathrm{~m}, 2 \mathrm{H}), 6.84-6.82(\mathrm{~m}, 2 \mathrm{H}), 5.29(\mathrm{t}, \mathrm{J}=3.5 \mathrm{~Hz}$, $1 \mathrm{H}), 3.97-3.92(\mathrm{~m}, 1 \mathrm{H}), 3.76(\mathrm{~s}, 3 \mathrm{H}), 3.61-3.58(\mathrm{~m}, 1 \mathrm{H}), 2.02-1.98(\mathrm{~m}, 1 \mathrm{H}), 1.87-1.83(\mathrm{~m}$, $2 \mathrm{H}), 1.69-1.60(\mathrm{~m}, 3 \mathrm{H}) \mathrm{ppm}$.

${ }^{13} \mathbf{C}$ NMR (CDCl 3 , 125 MHz): $\delta 154.9,151.5,118.2,114.9,97.7,62.4,56.0,30.9,25.7$, $19.3 \mathrm{ppm}$.

MS: $\mathrm{m} / \mathrm{z}=209[\mathrm{M}+\mathrm{H}]^{+}$.

2-Methoxy-4-methylphenyl tetrahydro-2H-pyran-2-yl ether (2i):

Reaction time: $3 \mathrm{~h}$.

Yield: $0.202 \mathrm{~g}(91 \%)$ as colourless oily liquid.

IR (neat) $\mathbf{v}_{\text {max }}: 3035,2941,1590,1511,912,871,812 \mathrm{~cm}^{-1}$.

${ }^{1} \mathbf{H}$ NMR $\left(\mathbf{C D C l}_{3}, \mathbf{5 0 0} \mathbf{M H z}\right): \delta 7.01(\mathrm{~d}, \mathrm{~J}=8.0 \mathrm{~Hz}, 1 \mathrm{H}), 6.72(\mathrm{~d}, \mathrm{~J}=1.5 \mathrm{~Hz}, 1 \mathrm{H}), 6.69(\mathrm{dt},$. $=8.0,1.0 \mathrm{~Hz}, 1 \mathrm{H}), 5.33(\mathrm{t}, \mathrm{J}=3.0 \mathrm{~Hz}, 1 \mathrm{H}), 4.05-4.00(\mathrm{~m}, 1 \mathrm{H}), 3.84(\mathrm{~s}, 3 \mathrm{H}), 3.60-3.57(\mathrm{~m}$, $1 \mathrm{H}), 2.30(\mathrm{~s}, 3 \mathrm{H}), 2.03-2.01(\mathrm{~m}, 1 \mathrm{H}), 1.94-1.93(\mathrm{~m}, 1 \mathrm{H}), 1.90-1.87(\mathrm{~m}, 1 \mathrm{H}), 1.69-1.60(\mathrm{~m}$, 3H) $\mathrm{ppm}$.

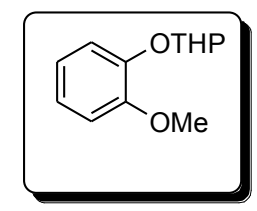


${ }^{13} \mathbf{C}$ NMR (CDCl ${ }_{3}, 125$ MHz): $\delta$ 150.1, 143.9, 132.3, 121.0, 118.2, 113.3, 97.8, 62.1, 55.9, 30.3, 25.2, 21.0, $18.8 \mathrm{ppm}$.

MS: $\mathrm{m} / \mathrm{z}=222[\mathrm{M}]^{+}$.

\section{4-Bromophenyl tetrahydro-2H-pyran-2-yl ether (2j):}

Reaction time: $3 \mathrm{~h}$.

Yield: $0.241 \mathrm{~g}(94 \%)$ as colourless oily liquid.

IR (neat) $\mathbf{v}_{\text {max }}: 3064,2943,1589,1578,919,823,645 \mathrm{~cm}^{-1}$.

${ }^{1} \mathbf{H}$ NMR $\left(\mathbf{C D C l}_{3}, \mathbf{5 0 0} \mathbf{M H z}\right): \delta 7.38-7.35(\mathrm{~m}, 2 \mathrm{H}), 6.95-6.92(\mathrm{~m}, 2 \mathrm{H}), 5.37(\mathrm{t}, \mathrm{J}=3.0 \mathrm{~Hz}$, $1 \mathrm{H}), 3.89-3.84(\mathrm{~m}, 1 \mathrm{H}), 3.61-3.57(\mathrm{~m}, 1 \mathrm{H}), 1.86-1.83(\mathrm{~m}, 2 \mathrm{H}), 1.72-1.63(\mathrm{~m}, 2 \mathrm{H}), 1.62-$ $1.57(\mathrm{~m}, 2 \mathrm{H}) \mathrm{ppm}$.

${ }^{13} \mathbf{C}$ NMR (CDCl 3 , 125 MHz): $\delta$ 156.1, 132.1, 118.2, 113.7, 96.4, 61.9, 30.1, 25.0, 18.5 ppm.

MS: $\mathrm{m} / \mathrm{z}=258[\mathrm{M}+2]^{+}$.

4-Chlorophenyl tetrahydro-2H-pyran-2-yl ether (2k):

Reaction time: $3 \mathrm{~h}$.

Yield: $0.191 \mathrm{~g}(90 \%)$ as colourless oily liquid.

IR (neat) $\mathbf{v}_{\max }: 3076,2944,1596,1583,1489,1021,965,919 \mathrm{~cm}^{-1}$.

${ }^{1} \mathbf{H}$ NMR $\left(\mathbf{C D C l}_{3}, \mathbf{5 0 0} \mathbf{~ M H z}\right): \delta$ 7.22-7.19 (m, 2H), 6.99-6.96 (m, 2H), $5.34(\mathrm{t}, \mathrm{J}=3.0$ $\mathrm{Hz}, 1 \mathrm{H}), 3.88-3.83(\mathrm{~m}, 1 \mathrm{H}), 3.60-3.56(\mathrm{~m}, 1 \mathrm{H}), 2.00-1.95(\mathrm{~m}, 1 \mathrm{H}), 1.86-1.82(\mathrm{~m}, 2 \mathrm{H})$, $1.68-1.56(\mathrm{~m}, 3 \mathrm{H}) \mathrm{ppm}$.

${ }^{13} \mathbf{C}$ NMR (CDCl 3 , $\left.125 \mathbf{M H z}\right): \delta$ 156.1, 129.7, 126.9, 118.3, 97.1, 62.4, 30.7, 25.6, $19.1 \mathrm{ppm}$.

MS: $\mathrm{m} / \mathrm{z}=214[\mathrm{M}+2]^{+}$.

4-Methoxyphenyl tetrahydro-2H-pyran-2-yl ether (2l):

Reaction time: $3 \mathrm{~h}$.

Yield: $0.199 \mathrm{~g}(96 \%)$ as colourless oily liquid.

IR (neat) $v_{\text {max }}: 3039,2943,2872,1506,969,920,827 \mathrm{~cm}^{-1}$.

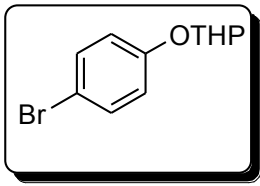

${ }^{1} \mathbf{H}$ NMR $\left(\mathbf{C D C l}_{3}, \mathbf{5 0 0} \mathbf{M H z}\right): \delta 7.19(\mathrm{dd}, \mathrm{J}=8.0,1.5 \mathrm{~Hz}, 1 \mathrm{H}), 6.98-6.95(\mathrm{~m}, 1 \mathrm{H}), 6.91-6.88(\mathrm{~m}, 2 \mathrm{H}), 5.38(\mathrm{t}, \mathrm{J}$ $=3.5 \mathrm{~Hz}, 1 \mathrm{H}), 4.04-3.99(\mathrm{~m}, 1 \mathrm{H}), 3.85(\mathrm{~s}, 3 \mathrm{H}), 3.61-3.58(\mathrm{~m}, 1 \mathrm{H}), 2.07-2.04(\mathrm{~m}, 1 \mathrm{H}), 1.95-1.94(\mathrm{~m}, 1 \mathrm{H}), 1.90-$ $1.88(\mathrm{~m}, 1 \mathrm{H}), 1.69-1.59(\mathrm{~m}, 3 \mathrm{H}) \mathrm{ppm}$.

${ }^{13} \mathbf{C}$ NMR (CDCl 3 , $\left.125 \mathbf{M H z}\right): \delta 154.4,151.0,117.7,114.4,97.2,61.9,55.5,30.4,25.2,18.8 \mathrm{ppm}$.

MS: $\mathrm{m} / \mathrm{z}=193[\mathrm{M}+\mathrm{H}]^{+}$.

4-Methylphenyl tetrahydro-2H-pyran-2-yl ether (2m):

Reaction time: $3 \mathrm{~h}$.

Yield: $0.180 \mathrm{~g}(94 \%)$ as colourless oily liquid.

IR (neat) $v_{\max }: 3027,2942,1609,1585,969,920,817 \mathrm{~cm}^{-1}$.

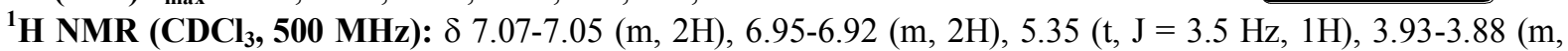
$1 \mathrm{H}), 3.59-3.55(\mathrm{~m}, 1 \mathrm{H}), 2.27(\mathrm{~s}, 3 \mathrm{H}), 2.01-1.96(\mathrm{~m}, 1 \mathrm{H}), 1.85-1.81(\mathrm{~m}, 2 \mathrm{H}), 1.67-1.55(\mathrm{~m}, 3 \mathrm{H}) \mathrm{ppm}$.

${ }^{13} \mathbf{C}$ NMR (CDCl 3 , 125 MHz): $\delta 154.9,130.8,129.7,116.4,96.5,61.9,30.4,25.2,20.4,18.8$ ppm.

MS: $\mathrm{m} / \mathrm{z}=193[\mathrm{M}+\mathrm{H}]^{+}$.

4-(Tetrahydro-2H-pyran-2-yloxy)benzaldehyde (2n):

Reaction time: $3 \mathrm{~h}$.

Yield: $0.187 \mathrm{~g}(91 \%)$ as colourless oily liquid.

IR (neat) $v_{\max }: 3050,2947,2756,1669,1602,966,834,787 \mathrm{~cm}^{-1}$.

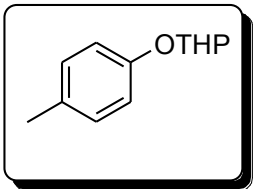

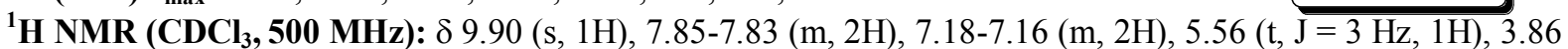
$(\mathrm{td}, \mathrm{J}=22.0,11.0,3.5 \mathrm{~Hz}, 1 \mathrm{H}), 3.66-3.63(\mathrm{~m}, 1 \mathrm{H}), 2.04-1.98(\mathrm{~m}, 1 \mathrm{H}), 1.92-1.89(\mathrm{~m}, 2 \mathrm{H}), 1.77-1.69(\mathrm{~m}, 2 \mathrm{H})$, $1.65-1.61(\mathrm{~m}, 1 \mathrm{H}) \mathrm{ppm}$.

${ }^{13} \mathbf{C}_{\text {NMR }}\left(\mathbf{C D C l}_{3}, \mathbf{1 2 5}\right.$ MHz): $\delta 190.9,162.1,131.7,130.4,116.4,96.0,62.0,30.0,24.9,18.3 \mathrm{ppm}$.

MS: $\mathrm{m} / \mathrm{z}=206[\mathrm{M}]^{+}$.

2-Methoxy-4-(1-propenyl)phenyl tetrahydro-2H-pyran-2-yl ether (2o):

Reaction time: $3 \mathrm{~h}$.

Yield: $0.225 \mathrm{~g}(91 \%)$ as colourless oily liquid.

IR (neat) $\mathbf{v}_{\max }: 3045,2950,1600,1591,965,927,882 \mathrm{~cm}^{-1}$.

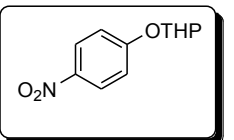

www.iosrjournals.org 
${ }^{1}$ H NMR $\left(\mathbf{C D C l}_{3}, \mathbf{5 0 0} \mathbf{M H z}\right): \delta 6.95(\mathrm{~d}, \mathrm{~J}=8.5 \mathrm{~Hz}, 1 \mathrm{H}), 6.79(\mathrm{bs}, 1 \mathrm{H}), 6.74(\mathrm{~d}, \mathrm{~J}=8.0 \mathrm{~Hz}, 1 \mathrm{H}), 6.23(\mathrm{~d}, \mathrm{~J}=$ $15.5 \mathrm{~Hz}, 1 \mathrm{H}), 6.05-5.98(\mathrm{~m}, 1 \mathrm{H}), 5.27(\mathrm{t}, \mathrm{J}=3.0 \mathrm{~Hz}, 1 \mathrm{H}), 3.92-3.87(\mathrm{~m}, 1 \mathrm{H}), 3.75(\mathrm{~d}, \mathrm{~J}=1.5 \mathrm{~Hz}, 3 \mathrm{H}), 3.50-3.47$ $(\mathrm{m}, 1 \mathrm{H}), 1.98-1.91(\mathrm{~m}, 1 \mathrm{H}), 1.85-1.83(\mathrm{~m}, 1 \mathrm{H}), 1.76(\mathrm{~d}, \mathrm{~J}=6.5 \mathrm{~Hz}, 3 \mathrm{H}), 1.58-1.50(\mathrm{~m}, 4 \mathrm{H}) \mathrm{ppm}$.

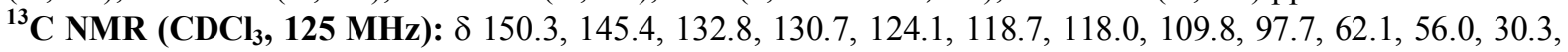
25.3, 18.8, $18.3 \mathrm{ppm}$.

HRMS (ESI) m/z Calcd for $\mathrm{C}_{15} \mathrm{H}_{21} \mathrm{O}_{3}(\mathrm{M}+\mathrm{H})^{+}, 249.1491$; Found, 249.1495.

\section{2-Methoxy-4-(2-propenyl)phenyl tetrahydro-2H-pyran-2-yl ether (2p):}

Reaction time: $3 \mathrm{~h}$.

Yield: $0.233 \mathrm{~g}(94 \%)$ as colourless oily liquid.

IR (neat) $v_{\max }: 3019,2941,1600,1581,962,917,871 \mathrm{~cm}^{-1}$.

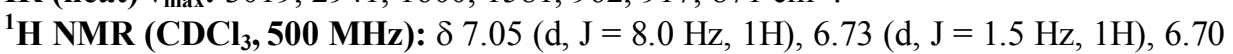
$(\mathrm{dd}, \mathrm{J}=8.0,1.5 \mathrm{~Hz}, 1 \mathrm{H}), 6.00-5.92(\mathrm{~m}, 1 \mathrm{H}), 5.35(\mathrm{t}, \mathrm{J}=3.0 \mathrm{~Hz}, 1 \mathrm{H}), 5.11-5.05(\mathrm{~m}$,

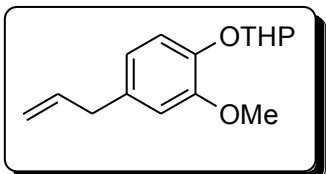
$2 \mathrm{H}), 4.04-3.99$ (m, 1H), 3.83 (s, 3H), 3.60-3.57 (m, 1H), 3.33 (d, J = 6.5 Hz, 2H), 2.03-1.86 (m, 3H), $1.66-1.61$ (m, 3H) ppm.

${ }^{13} \mathbf{C}$ NMR (CDCl ${ }_{3}, 125$ MHz): $\delta 150.6,144.9,137.9,134.8,121.0,118.5,115.9,113.2,98.1,62.4,56.3,40.2$, 30.7, 25.6, $19.2 \mathrm{ppm}$.

MS: $\mathrm{m} / \mathrm{z}=248[\mathrm{M}]^{+}$.

\section{1-Naphthyl tetrahydro-2H-pyran-2-yl ether (2q):}

Reaction time: $3 \mathrm{~h}$.

Yield: $0.221 \mathrm{~g}(97 \%)$ as colourless oily liquid.

IR (neat) $v_{\text {max }}: 3048,2942,1594,1579,958,917,872,792 \mathrm{~cm}^{-1}$.

${ }^{1}$ H NMR $\left(\mathbf{C D C l}_{3}, \mathbf{5 0 0}\right.$ MHz): $\delta 8.30-8.28(\mathrm{~m}, 1 \mathrm{H}), 7.76-7.74(\mathrm{~m}, 1 \mathrm{H}), 7.45-7.41(\mathrm{~m}, 3 \mathrm{H}), 7.33(\mathrm{t}, \mathrm{J}=8.0$ $\mathrm{Hz}, 1 \mathrm{H}), 7.11(\mathrm{~d}, \mathrm{~J}=8.0 \mathrm{~Hz}, 1 \mathrm{H}), 5.59(\mathrm{t}, \mathrm{J}=3.0 \mathrm{~Hz}, 1 \mathrm{H}), 3.90(\mathrm{td}, \mathrm{J}=7.0,6.0,3.0 \mathrm{~Hz}, 1 \mathrm{H}), 3.60-3.57$ $(\mathrm{m}, 1 \mathrm{H}), 2.13-2.10(\mathrm{~m}, 1 \mathrm{H}), 2.01-1.97(\mathrm{~m}, 1 \mathrm{H}), 1.93-1.89(\mathrm{~m}, 1 \mathrm{H}), 1.71-1.65(\mathrm{~m}, 2 \mathrm{H}), 1.59-1.56(\mathrm{~m}$, 1H) ppm.

${ }^{13} \mathbf{C}$ NMR (CDCl $\mathbf{3}, \mathbf{1 2 5}$ MHz): $\delta 152.9,135.0,127.9,126.5,126.4,126.4,125.5,122.3,121.4,108.6$, 96.7, 62.2, 30.9, 25.6, $19.2 \mathrm{ppm}$.

MS: $\mathrm{m} / \mathrm{z}=229[\mathrm{M}]^{+}$.

2-Naphthyl tetrahydro-2H-pyran-2-yl ether (2r):

Reaction time: $3 \mathrm{~h}$.

Yield: $0.216 \mathrm{~g}(95 \%)$ as colourless oily liquid.

IR (neat) $\mathbf{v}_{\max }: 3046,2949,1598,1585,965,924,882,798 \mathrm{~cm}^{-1}$.

${ }^{1} \mathbf{H}$ NMR $\left(\mathbf{C D C l}_{3}, \mathbf{5 0 0} \mathbf{M H z}\right): \delta$ 7.69-7.66 (m, 3H), $7.40(\mathrm{~d}, \mathrm{~J}=2.5 \mathrm{~Hz}, 1 \mathrm{H}), 7.35(\mathrm{td}, \mathrm{J}=8.0,1$ $\mathrm{Hz}, 1 \mathrm{H}), 7.26(\mathrm{td}, \mathrm{J}=7.0,1 \mathrm{~Hz}, 1 \mathrm{H}), 7.20(\mathrm{dd}, \mathrm{J}=9.0,2.5 \mathrm{~Hz}, 1 \mathrm{H}), 5.47(\mathrm{t}, \mathrm{J}=3.0 \mathrm{~Hz}, 1 \mathrm{H}), 3.89$ $3.84(\mathrm{~m}, 1 \mathrm{H}), 3.56-3.53(\mathrm{~m}, 1 \mathrm{H}), 1.98-1.93(\mathrm{~m}, 1 \mathrm{H}), 1.84-1.79(\mathrm{~m}, 2 \mathrm{H}), 1.63-1.54(\mathrm{~m}, 2 \mathrm{H}), 1.52-$ $1.47(\mathrm{~m}, 1 \mathrm{H}) \mathrm{ppm}$.

${ }^{13} \mathbf{C}$ NMR (CDCl 3 , 125 MHz): $\delta$ 155.1, 134.8, 129.7, 129.5, 127.9, 127.3, 126.5. 124.1, 119.4, 110.7, 96.6, 62.1, 30.6, 25.5, $19.0 \mathrm{ppm}$.

MS: $\mathrm{m} / \mathrm{z}=229[\mathrm{M}]^{+}$.

\section{References}

[1]. Greene, T. W.; Wuts, P. G. M. Protective Groups in Organic Synthesis, 2nd Ed. John Wiley \& Sons: New York, 1999.

[2]. (a)Corey, E. J.; Niwa, H.; Knolle, J. J. Am. Chem. Soc. 1978, 100, 1942-1943; (b) Bernady, K. F.; Floyd, M. B.; Poletto, J. F.; Weiss, M. J. J. Org. Chem. 1979, 44, 1438-1447.

[3]. Alper, H.; Dinkes, L. Synthesis 1972, 2,81.

[4]. Ramu, B. C.; Saha, M. J. Org. Chem. 1994, 59, 8269-8270.

[5]. Naik, S.; Gopinath, R.; Patel, B. K. Tetrahedron Lett. 2001, 42, 7679-7681.

[6]. Miyashita, M.; Yoshikoshi, A.; Grieco, P. A. J. Org. Chem. 1977, 42, 3772-3774.

[7]. Hon, Y. S.; Lee, C. F.; Chen, R. J.; Szu, P. H. Tetrahedron 2001, 57, 5991-6001.

[8]. Namboodiri, V. V.; Varma, R. S. Tetrahedron Lett. 2002, 43, 1143-1146.

[9]. Mineno, T. Tetrahedron Lett. 2002, 43, 7975-7978.

[10]. Stephens, J. R.; Butler, P. L.; Clow, C. H.; Oswald, M. C.; Smith, R. C.; Mohan, R. S. Eur. J. Org. Chem. 2003, 3827-3831.

[11]. Karimi, B.; Maleki, J. Tetrahedron Lett. 2002, 43, 5353-5355.

[12]. Namboodiri, V. V.; Verma, R. S. Chem. Commun. 2002, 342-343.

[13]. Yadav, J. S.; Reddy, B. V. S.; Gnaneshwar, D. New J. Chem. 2003, 27, 202-204.

[14]. Khan, A. T.; Mondal, E.; Borah, B. M.; Ghosh, S. Eur. J. Org. Chem. 2003, 4113-4117.

[15]. Khan, A. T.; Choudhury, L. H.; Ghosh, S. Tetrahedron Lett. 2004, 45, 7891-7894.

[16]. Pachamuthu, K.; Vankar, Y. D. J. Org. Chem. 2001, 66, 7511-7513.

[17]. Khan, A. T.; Ghosh, S.; Choudhury, L. H. Eur. J. Org. Chem. 2005, 4891-4896.

[18]. Oates, R. P.; Jones, P. B. J. Org. Chem. 2008, 73, 4743-4745.

[19]. a)Stevenson, C. P.; Nielsen, L. P. C.; Jacobsen, E. N. Organic Syntheses, 2006, 83, 162-169; (b) But, T. Y. S.; Lu, J.; Toy, P. H. Synlett 2010, 1115-1117.

[20]. P.O. Bishop, Neurophysiology of binocular vision, in J.Houseman (Ed.), Handbook of physiology, 4 (New York: Springer-Verlag, 1970) 342-366. (8) 